\title{
¿Cómo enseñar las matemáticas y ciencias experimentales? Resolviendo el dilema entre transmisión e indagación ${ }^{1}$
}

\author{
Juan D. Godino \\ jgodino@ugr.es \\ https://orcid.org/0000-0001-8409-0258 \\ Universidad de Granada \\ Granada, España \\ María Burgos \\ mariaburgos@ugr.es \\ https://orcid.org/0000-0002-4598-7684 \\ Universidad de Granada \\ Granada, España
}

Recibido: 24/03/2020 Aceptado: 28/04/2020

\section{Resumen}

A pesar de los enormes esfuerzos de investigación que se vienen realizando, el problema de cómo enseñar las matemáticas y las ciencias sigue abierto. Decidir entre los modelos didácticos centrados en el profesor (enseñanza transmisiva) o centrados en el estudiante (aprendizaje indagativo) plantea un dilema para la práctica educativa. En este trabajo abordamos este problema y proponemos una posible solución aplicando los supuestos y herramientas teóricas del Enfoque Ontosemiótico. Se argumenta que la optimización del aprendizaje y el logro de una acción didáctica idónea requiere entretejer de manera dialéctica y compleja los momentos de transmisión del conocimiento por el profesor con los momentos de indagación del estudiante. La implementación de trayectorias didácticas eficientes por parte del docente, implica la articulación de diversos tipos de configuraciones didácticas gestionadas mediante criterios de idoneidad, los cuales deben tener en cuenta las dimensiones epistémica, cognitiva, afectiva, interaccional y mediacional.

Palabras clave: Modelos didácticos, objetivismo, enfoque ontosemiótico, idoneidad didáctica.

\section{Como ensinar a matemática e as ciências experimentais? Resolvendo o dilema entre transmissão e indagação}

\begin{abstract}
Resumo
Apesar dos enormes esforços de investigação que se vêm realizando, o problema de como ensinar matemática e ciências permanece em aberto. Decidir ente um modelo didático centrado no professor (ensino transmissivo) ou um modelo didático centrado no estudante (aprendizagem indagativa), representa um dilema para a prática educacional. Neste trabalho, abordamos este problema e propomos uma solução aplicando os pressupostos teóricos e ferramentas do Enfoque Ontossemiótico. Argumenta-se que a otimização da aprendizagem e a realização de uma ação didática idónea requer um cruzamento dialético e complexo dos momentos de transmissão do conhecimento pelo professor com os momentos de indagação do estudante. A implementação de trajetórias didáticas eficientes implica por parte do professor a articulação de diversos tipos
\end{abstract}

\footnotetext{
${ }^{1}$ Versión ampliada en español de la conferencia invitada presentada en el CISETC, Arequipa, 2019.
} 
de configurações didáticas orientadas por critérios de idoneidade didática, os quais devem ter em consideração as dimensões epistêmica, cognitiva, afetiva e interacional.

Palavras chave: Modelos didáticos, objetivismo, enfoque ontossemiótico, idoneidade didática.

\title{
How to teach mathematics and experimental sciences? Solving the inquiring versus transmission dilemma
}

\begin{abstract}
Despite the huge research efforts that have been made, the problem of how to teach mathematics and sciences remains open. Deciding between teacher-focused teaching models (transmissive teaching) or student-focused (inquiring learning) poses a dilemma for educational practice. In this paper we address this problem and propose a solution applying the Onto-semiotic Approach assumptions and theoretical tools. We argue that the learning optimization and achievement of an appropriate didactic intervention require interweaving in a dialectical and complex way, the teacher's moments of knowledge transmission with the student's inquiry moments. The implementation of efficient didactic trajectories implies the articulation of diverse types of didactic configurations managed through didactical suitability criteria on the teacher's part. These should take into account the epistemic, cognitive, affective, interactional, mediational and ecological dimensions involved in instructional processes.
\end{abstract}

Keywords: didactical models, constructivism, objectivism, onto-semiotic approach, didactical suitability

\section{Introducción}

La investigación en didáctica de las matemáticas y las ciencias se interesa usualmente por describir y comprender los procesos de enseñanza y aprendizaje evitando proponer normas sobre cómo deberían ser dichos procesos. En las actividades de investigación predomina el componente científico descriptivo-explicativo frente al componente tecnológico de acción efectiva sobre la práctica educativa. No obstante, la intervención sobre los problemas reales de la clase requiere desarrollar teorías instruccionales específicas que ayuden al profesor en la toma de decisiones en las fases de diseño, implementación y evaluación. Se precisa elaborar teorías educativas que articulen las facetas epistémica y ecológica (teorías curriculares), con las facetas cognitiva y afectiva (teorías del aprendizaje), orientadas a la práctica de la enseñanza (teorías de diseño instruccional). En particular, la optimización de la faceta interaccional, esto es, los tipos de interacciones entre profesor y estudiantes, constituye un dilema: "Por una parte, la comunidad está repleta con afirmaciones que apoyan la enseñanza de las ciencias basada en la indagación, mientras que por otra parte hay un número considerable de preocupaciones y cuestiones que la desafían" (Zang, 2016, p. 898). 
En diversos trabajos venimos abordando la problemática del diseño instruccional en educación matemática y en ciencias experimentales desde el punto de vista del Enfoque Ontosemiótico del conocimiento y la instrucción matemáticos (EOS) (Godino, Batanero \& Font, 2007; Godino, Batanero \& Font, 2019). Utilizamos la noción de configuración ontosemiótica para mostrar la complejidad del conocimiento, ya que permite reconocer la trama de objetos y procesos que se ponen en juego en la actividad de resolución de problemas, la cual constituye la razón de ser de tales conocimientos. Así mismo, la noción de configuración didáctica ayuda a reconocer la variedad y dinámica de roles docentes y discentes implicada en los procesos instruccionales de los contenidos objeto del aprendizaje. Los diversos tipos de configuraciones didácticas (Godino, Contreras \& Font, 2006) deben ser articulados formando trayectorias didácticas cuya gestión por el profesor debe ser orientada por criterios de idoneidad (Godino, 2013; Breda, Font \& Pino-Fan, 2018).) a fin de lograr la eficiencia de la actividad educativa. En síntesis, la aplicación de las herramientas de análisis e intervención didáctica que propone el EOS permite construir una teoría de la instrucción orientada hacia la mejora progresiva de la práctica de la enseñanza de las matemáticas y las ciencias.

En este artículo ampliamos y revisamos la conferencia invitada presentada en el CISECT (Godino, 2019), incorporando en el hilo argumental de la misma ideas de trabajos previos sobre el problema de la articulación de modelos didácticos centrados en el profesor o en los estudiantes (Godino, Batanero, Cañadas \& Contreras, 2015; Godino, Rivas, Burgos \& Wilhelmi, 2018) que justifican la pertinencia de un modelo instruccional de tipo mixto. En este sentido, se describe un ejemplo que muestra la complejidad ontosemiótica de las prácticas matemáticas en el caso de la resolución de una tarea sobre proporcionalidad inversa y otro ejemplo de una experiencia con estudiantes de primaria, siguiendo el modelo de dialógico - colaborativo descrito en este trabajo como un primer encuentro con el concepto de proporcionalidad (Burgos \& Godino, 2019). Introducimos precisiones significativas relativas al tipo de configuración didáctica dialógica-colaborativa que consideramos idónea en las situaciones de primer encuentro del estudiante con un contenido nuevo, así como la pertinencia de aplicar el modelo didáctico en el campo de la educación en ciencias experimentales.

En la sección 2 describimos con más detalle el dilema existente entre dos posturas extremas sobre los tipos de interacciones didácticas que se deberían implementar en los procesos instruccionales: el constructivismo, con énfasis en la indagación y autonomía del estudiante, y 
el objetivismo que pone el acento en la transmisión del conocimiento. En la sección 3 introducimos un factor clave a tener en cuenta a la hora de decidir entre las dos posturas extremas: reconocer la complejidad ontosemiótica del conocimiento matemático y científico, la cual se debe tener cuenta, junto con otras razones cognitivas, para secuenciar y articular los procesos de transmisión e indagación. En la sección 4 describimos algunas herramientas del EOS para el análisis y diseño instruccional, las cuales son usadas para presentar en la sección 5 el modelo instruccional de tipo mixto que proponemos para optimizar la eficiencia de la actividad didáctica. Dicho modelo es explicado con un ejemplo de aplicación en la sección 6.

\section{Constructivismo versus objetivismo}

La familia de teorías instruccionales denominadas "Inquiry-Based Education” (IBE), “Inquiry-Based Learning” (IBL), "Problem-Based Learning” (PBL), postulan el aprendizaje basado en la indagación con poca guía por parte del profesor (Artigue \& Blomhøj, 2013). Las distintas variedades de constructivismo comparten, entre otros, los supuestos de que el aprendizaje es un proceso activo, que el conocimiento es construido en lugar de innato o pasivamente absorbido y que para lograr un aprendizaje efectivo es necesario el planteamiento a los estudiantes de problemas significativos, abiertos y desafiantes (Ernest, 1994; Fox, 2001).

El argumento de que los seres humanos son agentes activos que construyen el conocimiento por sí mismos ha hecho creer a las personas que las actividades instruccionales deberían estimular a los aprendices a construir el conocimiento a través de sus propias participaciones. Esta visión constructivista juega un papel importante en la enseñanza y aprendizaje de la ciencia y se ha convertido en un paradigma de enseñanza dominante. (Zhang, 2016, p. 897).

Las ideas sobre implementar una enseñanza y aprendizaje de las matemáticas y las ciencias basadas en la indagación han venido jugando un papel significativo en las orientaciones curriculares de diversos países, en proyectos, centros de investigación e iniciativas de reforma. Linn, Clark y Slotta (2003) definen el aprendizaje de las ciencias basado en la indagación como sigue:

Definimos indagación como comprometer a los estudiantes en el proceso intencional de diagnosticar problemas, criticar experimentos, distinguir alternativas, planificar investigaciones, revisar puntos de vista, explorar conjeturas, buscar información, construir modelos, debatir con los compañeros, comunicar a diversas audiencias, y elaborar argumentos coherentes. (Linn, Clark \& Slotta, 2003, p. 518). 
En los modelos pedagógicos que asumen los principios constructivistas, el papel del profesor debe ser elaborar un entorno de aprendizaje con el que el estudiante interactúe de manera autónoma. Esto significa que el profesor debe seleccionar cuidadosamente unas tareas de aprendizaje y asegurar que el estudiante disponga de los recursos cognitivos y materiales necesarios para implicarse en la solución de los problemas. Además, debe crear un andamiaje cognitivo, una "arquitectura de elecciones", que apoye y promueva la construcción del conocimiento por los propios estudiantes. En cierta manera se trata de implementar una pedagogía "paternalista libertaria" en el sentido de Thaler y Sunstein (2008), basada en el diseño de intervenciones del tipo "nudge". "Un nudge, según usaremos este término, es cualquier aspecto de la arquitectura de elección que modifica el comportamiento de las personas de una manera predecible sin prohibir ninguna opción o cambiar significativamente sus incentivos económicos" (Thaler \& Sunstein, 208, p. 6).

En el caso del aprendizaje matemático se considera esencial el uso de situaciones problemas (aplicaciones a la vida cotidiana, a otros campos del saber, o problemas internos a la propia disciplina) para que los estudiantes puedan dar sentido a las estructuras conceptuales que configuran las matemáticas como una realidad cultural. Estos problemas constituyen el punto de partida de la práctica matemática, por lo que la actividad de resolución de problema, su formulación, comunicación y justificación se consideran claves en el desarrollo de la capacidad de afrontar la solución de problemas no rutinarios. Este es el objetivo principal de la tradición denominada problem solving (Schoenfeld, 1992), cuyo énfasis se centra en la identificación de heurísticas y estrategias metacognitivas. También es objetivo de otros modelos teóricos como la Teoría de Situaciones Didácticas (TSD) (Brousseau, 1997) y la Educación Matemática Realista (RME) (Freudenthal, 1973; 1991).

No obstante, existen posturas contrapuestas al constructivismo, como es el caso de Mayer (2004), Kirschner, Sweller y Clark (2006) entre otros, que justifican mediante una extensa gama de investigaciones la mayor efectividad de modelos instruccionales en los cuales se atribuye al profesor y a la transmisión de conocimientos, un papel predominante. Estas posturas se relacionan ya con posturas filosóficas objetivistas (Jonassen, 1991), ya con la instrucción directa o la pedagogía basada en lecciones (Boghossian, 2006).

Sweller, Kirschner y Clark (2007) afirman que la investigación empírica del último medio siglo sobre este problema proporciona una abrumadora y clara evidencia de que una 
mínima guía durante la instrucción es significativamente menos efectiva y eficiente que una guía específicamente diseñada para apoyar el procesamiento cognitivo necesario para el aprendizaje. Resultados similares se reflejan en el meta-análisis realizado por Alfieri, Brooks, Aldrich y Tenenbaum (2011). En sus conclusiones, estos autores afirman, que, en general, los efectos de las tareas de descubrimiento no asistido parecen limitados, frente a las tareas de descubrimiento estimulado (enhanced discovery tasks). Las oportunidades para el aprendizaje constructivo pueden no presentarse cuando los alumnos se quedan sin guía.

Quizás los hallazgos de estos meta-análisis pueden ayudar a alejar el debate de los problemas sobre las formas de descubrimiento no guiadas hacia una discusión fructífera, y la realización de investigaciones empíricas sobre cómo se implementa mejor el andamiaje cognitivo, cómo proporcionar retroalimentación en el aula, cómo crear ejemplos trabajados para las diversas variedades de contenido, y cuándo se deben proporcionar formas directas de instrucción durante el aprendizaje. (Alfieri et al., 2011, p.13).

Para el objetivismo, en particular en su versión conductista, el conocimiento es públicamente observable y el aprendizaje consiste en la adquisición de ese conocimiento mediante la interacción entre estímulos y respuestas. Con frecuencia, la forma de condicionamiento usado para lograr conductas verbales deseables es mediante la instrucción directa. Se pueden aportar razones de tipo cognitivo a favor de aplicar un modelo didáctico basado en la transmisión de conocimientos (objetivismo) frente a los modelos basados en la construcción autónoma (constructivismo). Kirschner et al. (2006) señalan que las posiciones constructivistas, con una instrucción mínimamente guiada, contradicen la arquitectura de la cognición humana e imponen una pesada carga cognitiva que impide el aprendizaje:

Tenemos destreza en un área porque nuestra memoria a largo plazo contiene cantidades enormes de información relativa al área. Esa información nos permite reconocer rápidamente las características de una situación y nos indica, a menudo inconscientemente, qué hacer y cuándo hacerlo. (Kirschner, et al., 2006, p. 76).

Otras razones que rechazan las posiciones constructivistas provienen del punto de vista de la psicología cultural. Harris (2012) afirma que:

Las explicaciones del desarrollo cognitivo han descrito generalmente al niño como un científico independiente, quien recoge datos de primera mano y forma teorías sobre el mundo natural. Yo argumento que esta metáfora es inapropiada para dar cuenta del aprendizaje cultural de los niños. En dicho dominio, los niños actúan más bien como antropólogos 
que atienden a, colaboran con, y aprenden de los miembros de su cultura. (Harris, 2012, p. 259).

La metáfora del niño como científico natural, es útil cuando se usa para describir cómo los niños dan sentido a las regularidades universales del mundo natural, regularidades que ellos pueden observar por sí mismos, sin importar cuál sea su entorno cultural. Sin embargo, la metáfora es engañosa si se utiliza para explicar de forma comprehensiva y global el desarrollo cognitivo. Los niños nacen en un mundo cultural que media sus encuentros con el mundo físico y biológico. Para acceder a dicho mundo cultural, los niños necesitan un modo de aprendizaje orientado socialmente (aprendizaje mediante la observación participante). "El dominio de regularidades normativas requiere aprendizaje cultural” (Harris, 2012, p. 269).

El debate entre la enseñanza directa, ligada a posiciones objetivistas sobre el conocimiento matemático y científico, que defiende un papel central del profesor para guiar el aprendizaje, y la enseñanza mínimamente guiada, usualmente referida al modelo de enseñanza de tipo constructivista, no está claramente resuelto en la literatura de investigación. HmeloSilver, Duncan y Chinn, (2007) argumentan que PBL e IBL "no son aproximaciones instruccionales mínimamente guiadas, sino que proporcionan un extenso soporte y una guía para facilitar el aprendizaje de estudiante" (p. 91). Los partidarios del aprendizaje basado en problemas y la indagación centran sus argumentos en la cantidad de guía y la situación en la que tal pauta se proporciona. Consideran que la guía que se da contiene un extenso cuerpo de apoyo y al estar inmersa en situaciones de la vida real ayuda a los estudiantes a dar sentido al contenido científico.

Para Zhang (2016) la tensión entre estas dos posiciones instruccionales no está en si una u otra es participaría de presentar más o menos guía o apoyo a los estudiantes, sino entre presentar explícitamente las soluciones a los aprendices o dejar que ellos las descubran. "Para los partidarios de la instrucción directa, la presentación explícita de las soluciones y la demostración del proceso para lograr las soluciones son una guía esencial” (p. 908). Pretender que los estudiantes descubran, exploren y encuentren las soluciones, según se estructura en IBE, elimina la necesidad de presentar las soluciones. En las posiciones constructivistas, aunque se admita una cierta dosis de transmisión de información del profesor al estudiante, sigue siendo esencial ocultar una parte del contenido. Para los partidarios de la instrucción directa que asumen la teoría de la carga cognitiva con énfasis en los ejemplos trabajados, proporcionar las 
soluciones se considera esencial. Por ejemplo, en el campo del desarrollo del razonamiento proporcional, Bentley y Yates (2017) usaron el modelo didáctico de "worked examples" para presentar la estrategia de reducción a la unidad, esto es, ayudar a los estudiantes a adoptar un análisis paso a paso de problemas de valor faltante mediante el cual reconocer fácilmente, en primer lugar, una unidad y después utilizarla para resolver el problema. En su investigación informan de resultados positivos cuando se aplica esta estrategia didáctica tanto para estudiantes con alto estatus socioeconómico como bajo.

En la siguiente sección vamos a introducir una nueva variable en el debate de los modelos didácticos basados en el constructivismo (indagativos) y el objetivismo (transmisivos). Se trata de reconocer la complejidad ontosemiótica del conocimiento matemático y científico, la cual debe ser tenida en cuenta en los procesos instruccionales cuando se pretende alcanzar el objetivo de optimizar los aprendizajes de los estudiantes. Al asumir presupuestos antropológicos, semióticos y pragmatistas sobre el conocimiento matemático se llega a la conclusión de que una parte esencial del conocimiento que tienen que aprender los estudiantes son reglas conceptuales, proposicionales, procedimentales, convenidas en el seno de la comunidad de prácticas matemáticas o científicas. Los estudiantes, para resolver los problemas que constituyen el objetivo educativo, parten de unos conocimientos previos, que en una parte central de los mismos son reglas, las cuales deben estar disponibles para comprender y abordar la tarea. No tiene sentido pretender que los estudiantes descubran esas reglas. Pero además el objetivo es encontrar las soluciones, que a su vez son nuevas reglas, las cuales deberán formar parte de su acervo cognitivo para resolver futuros problemas. Al tener en cuenta la complejidad ontosemiótica del conocimiento matemático y científico, sin dejar de reconocer el papel central de la resolución de problemas como razón de ser de los contenidos, se derivan los presupuestos de un modelo educativo-instruccional que se presenta como solución al dilema entre indagación y transmisión.

\section{Complejidad ontosemiótica del conocimiento matemático}

Los presupuestos ontosemióticos, epistemológicos y cognitivos del EOS (Godino et al., 2007, Godino et al., 2019) sirven de base para una propuesta educativa-instruccional. Aunque esta modelización del conocimiento se ha desarrollado y aplicado para el caso de las matemáticas se considera también pertinente para el núcleo central (conceptos y principios) del conocimiento científico. 
El EOS reconoce un papel clave a la transmisión (contextualizada y significativa para el estudiante) de conocimientos en los procesos de enseñanza de las matemáticas, sin que la resolución de problemas y la indagación dejen de tener un papel importante en el proceso de aprendizaje. Se trata de tener en cuenta la naturaleza cultural/regulativa de los objetos implicados en las prácticas matemáticas cuya realización competente por los alumnos se pretende. Esta competencia no se puede considerar como adquirida si carece de sentido para los estudiantes y, por lo tanto, se requiere que sea inteligible y significativa para ellos. Así, los estudiantes deben ser capaces de utilizar los objetos matemáticos en contextos propios con autonomía. Pero, según el EOS, debido a la complejidad ontosemiótica del conocimiento matemático, esta autonomía no se debe adquirir necesariamente en el primer encuentro con el objeto o en la determinación de algunos de los sentidos que se le atribuyan; por ejemplo, se puede alcanzar en una práctica matemática de aplicación.

El EOS entiende las matemáticas como una actividad de las personas implicadas en la solución de cierta clase de situaciones - problemas, e interpreta el significado institucional y personal de los objetos matemáticos en términos de los subsistemas de prácticas institucionales y personales ligadas a contextos de uso y de objetos emergentes (situaciones, lenguajes, conceptos, proposiciones, procedimientos y argumentos). La progresión en el aprendizaje tiene lugar a medida que el sujeto se apropia de los diversos significados, y reconoce y comprende la trama de objetos implicados en los mismos. Esto es, el estudiante se debe apropiar de las prácticas matemáticas institucionales y de los objetos y procesos implicados en la resolución de las situaciones - problemas cuyo aprendizaje se pretende (Figura 1).

En un proceso de instrucción, la realización por el alumno de las prácticas matemáticas ligadas a la solución de ciertas tareas problemáticas pone en juego un conglomerado de objetos y procesos cuya naturaleza, desde el punto de vista institucional es esencialmente normativa (Font, Godino y Gallardo, 2013). No parece necesario ni posible que los estudiantes tengan que descubrir de manera autónoma las convenciones que determinan estos objetos que han emergido tras un proceso histórico y cultural orientado hacia la generalización, formalización y maximización de la eficiencia del trabajo matemático. 
Figura 1. Significados pragmáticos y configuración ontosemiótica

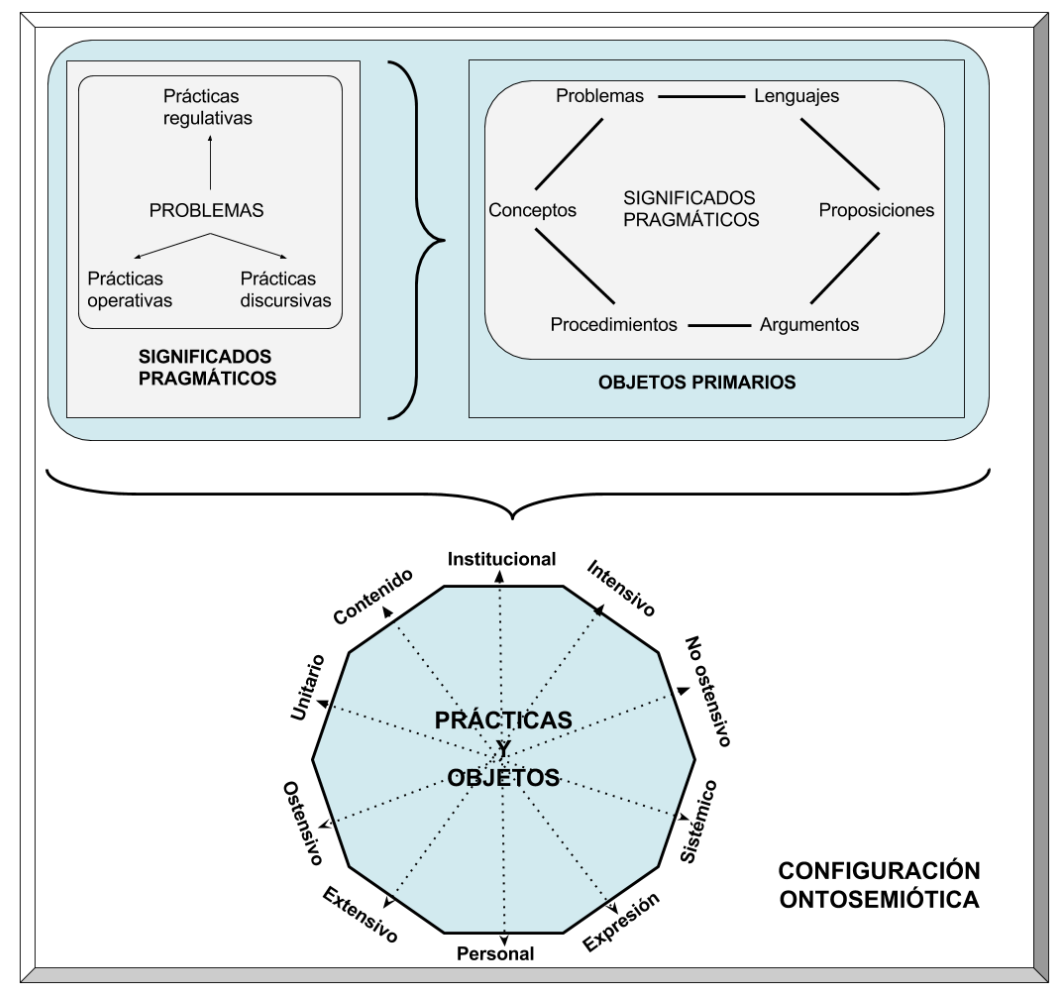

Fuente: Godino et al (2017)

En la ontología matemática del EOS, en consonancia con la filosofía de la matemática de Wittgenstein (Baker \& Hacker, 1985; Bloor, 1983; Wittgenstein, 1953, 1976), los conceptos, proposiciones y procedimientos son concebidos como reglas gramaticales de los lenguajes que se usan para describir nuestros mundos. No describen propiedades de unos objetos que tengan algún tipo de existencia independiente de las personas que los construyen o inventan, ni de los lenguajes mediante los cuales se expresan. El tipo de existencia de los conceptos, de las proposiciones y los procedimientos de las configuraciones epistémicas es el que tienen las reglas convencionales. Desde esta perspectiva, la verdad o necesidad matemática no es más que el estar de acuerdo con el resultado de seguir una regla que forma parte de un juego de lenguaje que se pone en funcionamiento en determinadas prácticas sociales. No es un acuerdo de opiniones arbitrarias, es un acuerdo de prácticas sometidas a reglas.

La realización de las prácticas matemáticas supone la intervención de objetos previos para comprender las demandas de la situación - problema y poder implementar una estrategia de partida. Tales objetos, sus reglas y condiciones de aplicación, deben estar disponibles en la memoria de trabajo del sujeto. Aunque sea posible buscar tales conocimientos por sí mismo en 
el espacio de trabajo, no siempre hay suficiente tiempo o el alumno no lo logra; por ello, el profesor y los compañeros pueden prestar un apoyo inestimable para evitar la frustración y el abandono.

Seguidamente ejemplificamos el uso de la herramienta configuración ontosemiótica para el caso del objeto matemático proporcionalidad, contextualizado con una tarea sobre proporcionalidad inversa (Figura 2) usada en el contexto de formación de profesores. Se pretende revelar la complejidad del aprendizaje de este objeto matemático, poniendo en discusión la pertinencia de abordar dicho estudio de forma global mediante un modelo didáctico constructivista, o con un modelo basado en la transmisión de información.

La tarea que presentamos puede ser significativa para los estudiantes, ya que la bicicleta presentada es un objeto auténtico tomado de un museo y se puede aprender algo de historia de la descripción del proceso de invención científica, junto con los principios físicos y sus justificaciones matemáticas. Pone en juego, por tanto, una enseñanza interdisciplinar que combina las matemáticas con la historia y la ciencia.

Figura 2. Proporcionalidad inversa

1) Si la longitud de la circunferencia delantera (grande) de la bicicleta es $462 \mathrm{~cm}$ y la de la trasera (pequeña) es de $132 \mathrm{~cm}$, ¿qué distancia debe recorrer la bicicleta para que la rueda pequeña de 30 vueltas más que la grande.

2) Encuentra una explicación matemática del movimiento de la bicicleta. ¿Cómo lo explicarías a tus estudiantes?

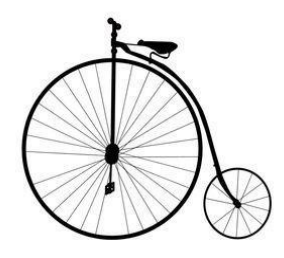

Fuente: adaptado de Ben-Chain, Keret, e Ilany, 2012, p.187)

La tarea se puede resolver de diferentes maneras que involucran diversos niveles de algebrización (Godino, Aké, Gonzato y Wilhelmi, 2014; Godino, Neto, Wilhelmi, Aké, Etchegaray y Lasa, 2015). El reconocimiento de estos niveles de algebrización en las prácticas matemáticas ayuda a comprender las dificultades de aprendizaje y planificar la enseñanza, ya que tienen en cuenta los progresivos grados de generalización de los objetos que se ponen en juego, el cálculo analítico que se aplica a los mismos y el tipo de lenguaje usado. Se distingue la actividad matemática puramente aritmética (nivel 0 de algebrización) de progresivos niveles de algebrización, según los siguientes criterios (Godino et al, 2015, p. 135):

- Nivel 1. Se usan objetos intensivos (generales) de segundo grado de generalidad, propiedades de la estructura algebraica de $N$ y la igualdad como equivalencia. 
- Nivel 2. Se usan representaciones simbólico - literales para referir a los objetos intensivos reconocidos, los cuales están ligados a la información espacial, temporal y contextual; se resuelven ecuaciones de la forma $\mathrm{A} x+\mathrm{B}=\mathrm{C}(\mathrm{A}, \mathrm{B}, \mathrm{C} \in R)$.

- Nivel 3. Los símbolos se usan de manera analítica, sin referir a la información contextual. Se realizan operaciones con indeterminadas o variables; se resuelven ecuaciones de la forma $A x+B=C x+D(\mathrm{~A}, \mathrm{~B}, \mathrm{C}, \mathrm{D} \in R)$.

- Nivel 4. Se estudian familias de ecuaciones y funciones usando parámetros y coeficientes.

- Nivel 5. Se realizan cálculos analíticos (sintácticos) que implican el uso de uno más parámetros, junto con variables o indeterminadas.

- Nivel 6. Se comienza a estudiar estructuras algebraicas en sí mismas, sus definiciones y propiedades estructurales.

En Cuadro 1 (columna 1) se incluye una secuencia de prácticas elementales para resolver el problema de la Figura 2 que ponen en juego el nivel 3 de algebrización; en la columna 2 se indica el uso e intencionalidad de cada una de las prácticas, mientras que en la columna 3 se especifican los objetos referidos en las prácticas (conceptos, proposiciones, procedimientos y argumentos).

Cuadro 1. Configuración ontosemiótica de una solución de nivel 3 de algebrización

\begin{tabular}{|l|l|l|}
\hline $\begin{array}{l}\text { Secuencia de prácticas } \\
\text { elementales para resolver } \\
\text { la tarea }\end{array}$ & $\begin{array}{l}\text { Uso e intencionalidad de } \\
\text { las prácticas }\end{array}$ & $\begin{array}{l}\text { Objetos referidos en las prácticas } \\
\text { (conceptos, proposiciones, procedimientos, } \\
\text { argumentos. })\end{array}$ \\
\hline $\begin{array}{l}\text { 1) En el desplazamiento de } \\
\text { la bicicleta, la distancia } \\
\text { que recorren las dos ruedas } \\
\text { es la misma. }\end{array}$ & $\begin{array}{l}\text { Explicitar las } \\
\text { características físicas de la } \\
\text { situación (movimiento de } \\
\text { una bicicleta) }\end{array}$ & $\begin{array}{l}\text { Conceptos: magnitud continua longitud; } \\
\text { desplazamiento, distancia. } \\
\text { Proposición } \text { P1: Enunciado de la práctica 1) } \\
\text { Argumento: rigidez física del objeto en } \\
\text { movimiento (la bicicleta) }\end{array}$ \\
\hline $\begin{array}{l}\text { 2) Se puede obtener como } \\
\text { el producto de la longitud } \\
\text { de la circunferencia de una } \\
\text { de las ruedas por el } \\
\text { número de vueltas que da } \\
\text { ésta. }\end{array}$ & $\begin{array}{l}\text { Establecer la relación } \\
\text { entre las magnitudes que } \\
\text { intervienen en el } \\
\text { problema. }\end{array}$ & $\begin{array}{l}\text { Conceptos: magnitud continua longitud; } \\
\text { magnitud discreta número de vueltas; } \\
\text { circunferencia } \\
\text { Proposición } \text { P2: Enunciado de la práctica 2) } \\
\text { (D = Lxn) } \\
\text { Argumento: condiciones que describen el } \\
\text { movimiento de una rueda }\end{array}$ \\
\hline
\end{tabular}

Continúa... 
Cuadro 1. Configuración ontosemiótica de una solución de nivel 3 de algebrización (Cont.)

\begin{tabular}{|c|c|c|}
\hline $\begin{array}{l}\text { 3) Llamemos } x \text { al número } \\
\text { de vueltas que da la rueda } \\
\text { delantera. Se cumple que, } \\
462 x=132(x+30)\end{array}$ & $\begin{array}{l}\text { - Representar con un } \\
\text { símbolo literal el valor de } \\
\text { la incógnita. } \\
\text { - Relacionar la incógnita } \\
\text { con los datos del problema }\end{array}$ & $\begin{array}{l}\text { Proposición } \mathrm{P} 3 \text { : Las dos ruedas recorren la } \\
\text { misma distancia } \\
\text { Argumento: por la rigidez del cuerpo físico } \\
\text { que se desplaza. }\end{array}$ \\
\hline $\begin{array}{l}\text { 4) Esto es } 462 x= \\
132 x+3960 ; \text { de donde, } \\
462 x-132 x=3960, \\
y \text { finalmente } \\
x=3960 \div 330=12 .\end{array}$ & $\begin{array}{l}\text { - Despejar el valor de la } \\
\text { incógnita }\end{array}$ & $\begin{array}{l}\text { Procedimiento: despeje de la incógnita. } \\
\text { Argumento: propiedades aritméticas }\end{array}$ \\
\hline $\begin{array}{l}\text { 5) La bicicleta tendrá que } \\
\text { dar } 12 \text { vueltas y por tanto } \\
\text { recorrerá, } 462 \times 12= \\
5.544 \mathrm{~cm} .\end{array}$ & $\begin{array}{l}\text { Interpretar el resultado } \\
\text { numérico como solución } \\
\text { al problema }\end{array}$ & $\begin{array}{l}\text { Proposición } \mathrm{P} 4: \text { La bicicleta dará } 12 \text { vueltas } \\
\text { y recorrerá } 5.544 \mathrm{~cm} \\
\text { Argumento: Secuencia de prácticas 1) a 5) }\end{array}$ \\
\hline
\end{tabular}

Fuente: Elaborado por los autores

Se pueden plantear variantes del enunciado del problema cuya solución involucre prácticas matemáticas de tipo aritmético (nivel 0 de algebrización), proto-algebraicas (niveles 1 y 2), o incluso prácticas propias de niveles superiores de algebrización que involucren el uso de parámetros (nivel 4). De esta manera se pone de manifiesto la riqueza de la situación, así como la variedad de conocimientos y competencias matemáticas que se ponen en juego. No parece, por tanto, pertinente que el proceso de aprendizaje de la resolución de tareas que involucran la proporcionalidad simple (directa, inversa) y compuesta, así como sus diversos significados se pueda lograr mediante la aplicación de modelos didácticos centrados en el descubrimiento autónomo de los estudiantes. En el diseño de los procesos de instrucción es importante tener en cuenta los diversos significados de la proporcionalidad (Burgos y Godino, 2020), los cuales deben tener lugar en un dilatado espacio de tiempo (educación primaria, secundaria, universidad) y en distintas áreas de contenido (Wilhelmi, 2017).

\section{Herramientas del EOS para el análisis didáctico de diseños instruccionales}

En Godino et al. (2006) se desarrollan algunas herramientas teóricas para el análisis de los procesos de instrucción teniendo en cuenta el modelo ontosemiótico sobre el conocimiento matemático previamente desarrollado (Godino et al, 2007; Font et al., 2013). En particular, las nociones de configuración didáctica e idoneidad didáctica sirven como base para definir un 
modelo didáctico mixto que articula los procesos de indagación y transmisión del conocimiento, relacionados de manera dialéctica en diferentes tipos de configuraciones didácticas.

\section{Configuración didáctica}

Una configuración didáctica es cualquier segmento de actividad matemática en un proceso de enseñanza y aprendizaje, que queda comprendido entre el inicio y fin de una tarea (situación - problema). Así, cada segmento incluye tanto las acciones de los estudiantes y del profesor como los medios usados para abordar la tarea. La secuencia de configuraciones didácticas constituye una trayectoria didáctica. La trayectoria didáctica incluye como uno de sus componentes a la "trayectoria hipotética de aprendizaje" (Simon, 1995; Simon \& Tzur, 2004), ya que se tiene en cuenta no solo los objetivos, tareas instruccionales e hipótesis sobre el proceso de aprendizaje, sino también los roles docentes y discentes y los medios instruccionales empleados.

La tarea que delimita una configuración didáctica puede estar formada por distintas subtareas cada una de las cuales se puede considerar como una subconfiguración. Además, tanto las configuraciones como las subconfiguraciones didácticas pueden ser descritas y analizadas haciendo referencia a las dimensiones epistémica, instruccional y cognitivo-afectiva:

a) Configuración epistémica: sistema de prácticas, objetos y procesos matemáticos institucionales requeridos en la tarea.

b) Configuración instruccional: sistema de funciones docentes, discentes y medios instruccionales que se utilizan, así como las interacciones entre los distintos componentes.

c) Configuración cognitiva - afectiva: sistema de prácticas, objetos y procesos matemáticos personales que describe el aprendizaje y los componentes afectivos que le acompañan.

La Figura 3 resume los componentes y dinámica interna de las configuraciones didácticas, las relaciones entre la enseñanza y el aprendizaje y los principales procesos matemáticos ligados a la modelización ontosemiótica del conocimiento matemático (Godino, Font, Wilhelmi y Lurduy, 2011; Font, Godino y Gallardo, 2013). Dicha modelización, tiene en cuenta la complejidad de las relaciones que se establecen en el seno de cualquier configuración didáctica, no reducible a momentos exclusivamente indagatorios o únicamente de transmisión de conocimientos. 
Figura 3. Componentes y dinámica interna de una configuración didáctica

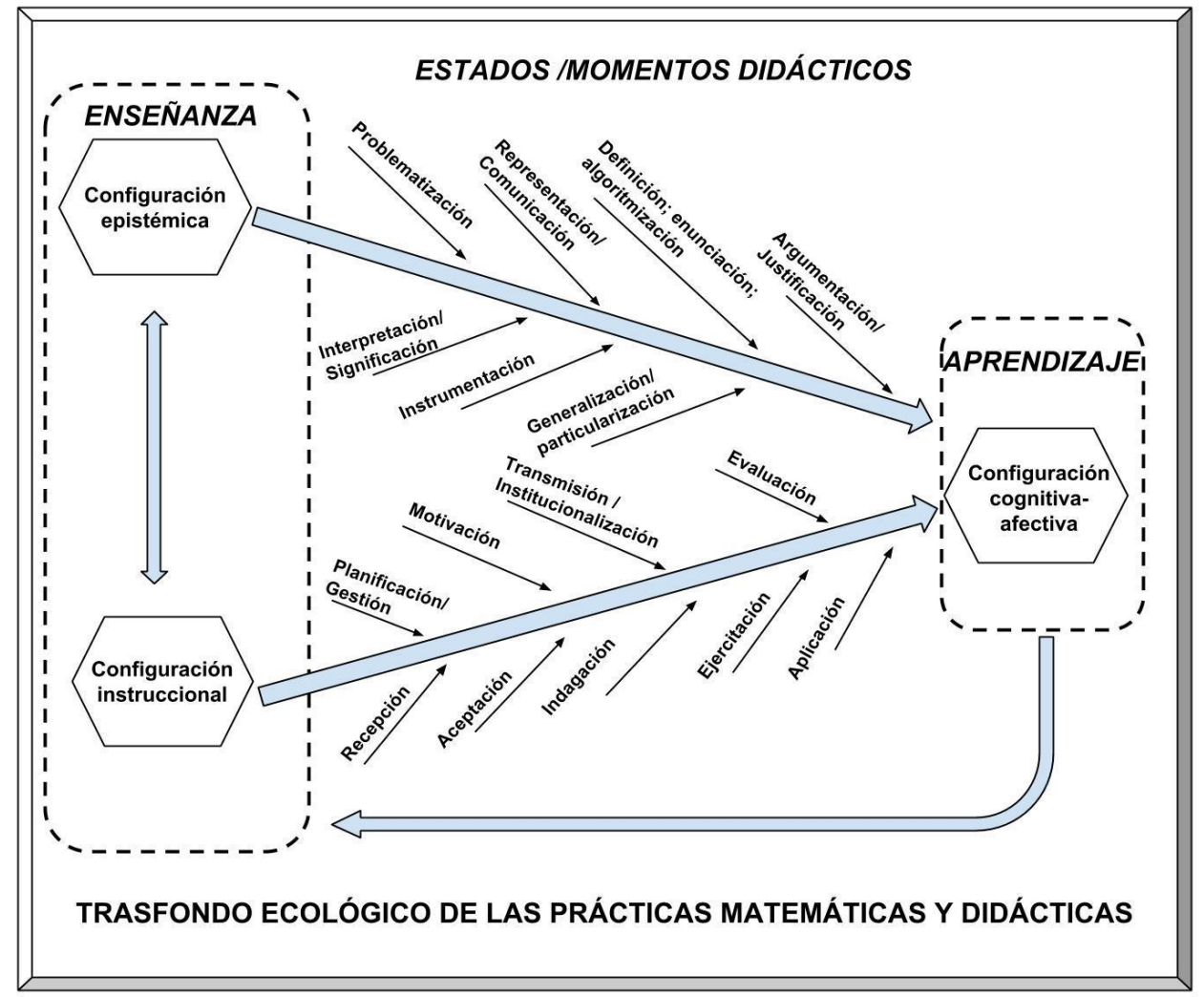

Fuente: Godino et al. (2015, p. 2646)

En la Figura 3, con la flecha inferior, del aprendizaje a la enseñanza, se quiere señalar que las relaciones no son lineales, sino cíclicas. En un momento de indagación, por ejemplo, el sujeto discente interacciona con la configuración epistémica sin intervención del docente (o con una influencia menor). Esta interacción condiciona las intervenciones docentes, que deben estar entonces previstas en la configuración instruccional, quizás no totalmente en su contenido, pero sí en su naturaleza, necesidad y utilidad. Esto, evidentemente, no es privativo de los momentos indagatorios. La trayectoria cognitiva produce ejemplos, significados, argumentos, etc., que condicionan el proceso de estudio y, en consecuencia, las configuraciones epistémica e instruccional.

\section{Idoneidad didáctica}

La idoneidad didáctica se define como el grado en que un proceso de instrucción (o una parte del mismo) reúne ciertas características que permiten calificarlo como óptimo o adecuado para conseguir la adaptación entre los significados personales logrados por los estudiantes 
(aprendizaje) y los significados institucionales pretendidos (enseñanza), teniendo en cuenta las circunstancias y recursos disponibles (entorno). Esto supone la articulación coherente y sistémica de seis criterios relativos a las facetas que intervienen en un proceso de instrucción (Godino et al., 2007, p. 133):

- Idoneidad epistémica, expresa el grado de representatividad de los significados institucionales implementados, respecto de un significado de referencia.

- Idoneidad ecológica, referida al grado en que el proceso de estudio se ajusta al proyecto educativo del centro, la escuela y la sociedad y a los condicionamientos del entorno en que se desarrolla.

- Idoneidad cognitiva, refiere al grado en que los significados implementados estén en la zona de desarrollo potencial de los alumnos, así como la proximidad de los significados personales logrados a los significados pretendidos.

- Idoneidad afectiva, expresa el grado de implicación (interés, motivación, etc.) del alumnado en el proceso de instrucción.

- Idoneidad interaccional, expresa el grado en que los tipos de configuraciones didácticas implementadas y su articulación permiten identificar y resolver los conflictos semióticos potenciales que se producen durante el proceso de instrucción.

- Idoneidad mediacional, grado de disponibilidad y adecuación de los recursos materiales y temporales necesarios para el desarrollo del proceso de enseñanza aprendizaje.

La noción de idoneidad didáctica y sus seis facetas proporcionan criterios para delimitar la significatividad de los hechos didácticos que acontecen en los procesos de instrucción matemática. Un hecho didáctico se considera significativo si aporta información empírica relevante sobre el desarrollo del proceso que se corresponda con alguno de los criterios de idoneidad previamente establecidos en alguna de las facetas. El logro de una alta idoneidad didáctica requiere un equilibrio entre los diferentes criterios parciales relativos a las distintas facetas, teniendo en cuenta el contexto en que tiene lugar. Es relativamente fácil conseguir alguno de los criterios por separado, pero lo que es más difícil y valioso es conseguir un cierto equilibrio entre los mismos.

La idoneidad es relativa a unas circunstancias temporales y contextuales cambiantes, lo que requiere una actitud de reflexión e investigación por parte del profesor y demás agentes que comparten la responsabilidad del proyecto educativo. Implica la asunción de una racionalidad 
axiológica en educación matemática que permita el análisis, la crítica, la justificación de la elección de los medios y de los fines, la justificación del cambio, y en definitiva responder a la pregunta genérica, ¿sobre qué aspectos se puede incidir para la mejora progresiva de los procesos de instrucción matemática? (Godino, Batanero \& Font, 2019).

\section{Un modelo instruccional mixto transmisivo - indagativo}

En los momentos o fases de primer encuentro del estudiante con un significado específico de un objeto $O$ se considera que una configuración dialógica - colaborativa puede optimizar el aprendizaje. En este tipo de configuración el docente y estudiantes trabajan juntos en la solución de problemas que ponen en juego el conocimiento $O$ de manera crítica; el primer encuentro debería apoyarse, por tanto, en una intervención experta del docente. El proceso de enseñanza-aprendizaje podría lograr de este modo mayor idoneidad epistémica y ecológica (Godino, Font, Wilhelmi \& Castro, 2009). En las fases de primer encuentro, un modelo didáctico indagativo, con mínima guía docente, los estudiantes están expuestos al riesgo de no encontrar las soluciones. "Aunque los estudiantes encuentren las soluciones por sí mismos, no conocen los procedimientos más efectivos ya que deben tantear sin rumbo fijo en el proceso de indagación del problema, sin mencionar la carga cognitiva que esto impone” (Zhang, 2016, p. 909).

Cuando las reglas y las circunstancias de aplicación que caracterizan el objeto de aprendizaje $\mathrm{O}$ sean comprendidas se puede tender hacia niveles mayores de idoneidad cognitiva y afectiva proponiendo profundizar en el estudio de $\mathrm{O}$ (situaciones de ejercitación y aplicación), mediante configuraciones didácticas que atribuyan progresivamente y de forma controlada mayor autonomía al estudiante (Figura 4). 
Figura 4. Modelo didáctico mix to indagativo-transmisivo

MAG: Magistral

PER: Personal

COL: Colaborativa DIA: Dialógica
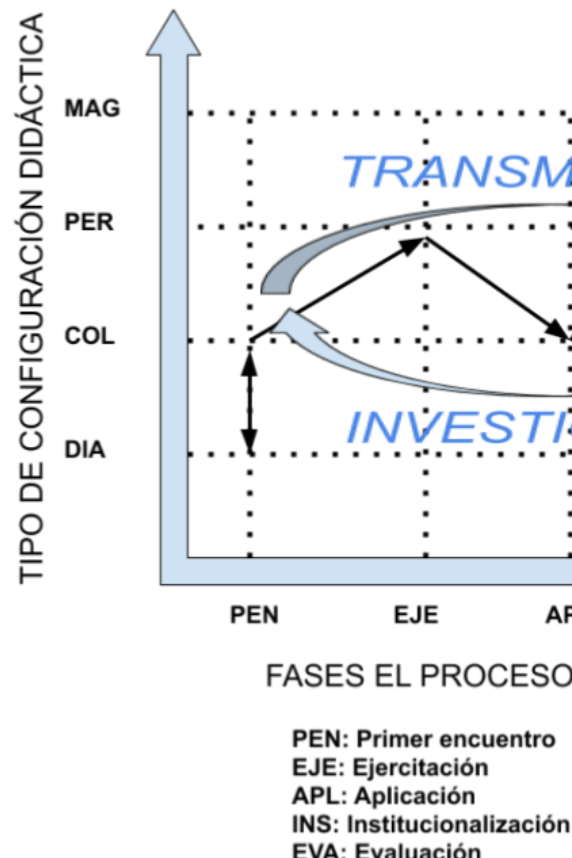

Fuente: Elaborada por los autores

En síntesis, en el marco del EOS, se asume que los tipos de configuraciones didácticas que promueven el aprendizaje pueden ser diversos dependiendo de los tipos de conocimientos pretendidos, del estado inicial del conocimiento de los sujetos, del contexto y circunstancias del proceso instruccional. Las configuraciones didácticas indagativas (constructivistas), colaborativas y transmisivas (magistrales) pueden tener lugar de manera secuencial, aunque sin orden rígido preestablecido. Cuando se trata del aprendizaje de un contenido nuevo y complejo la transmisión de conocimientos en momentos específicos, ya por parte del profesor, ya por el alumno líder en el seno de los equipos de trabajo, puede ser crucial en el proceso de aprendizaje. Esa transmisión puede ser significativa cuando los estudiantes están participando de la actividad y trabajando colaborativamente. La herramienta configuración didáctica ayuda a comprender la dinámica y complejidad de las interacciones entre el contenido, el docente, discentes y el medio. La optimización del aprendizaje puede tener lugar localmente mediante un modelo mix to que articula la transmisión de conocimientos, la indagación y la colaboración, modelo gestionado mediante criterios de idoneidad didáctica (Godino et al, 2007; Breda, Font \& Pino-Fan, 2018) interpretados y adaptados al contexto por el profesor. 
Algunas teorías didácticas, como la Teoría de la Objetivación (Radford, 2006; 2014) defienden de manera general la aplicación de un modelo colaborativo, trabajo conjunto de profesor y estudiantes, como preferible a las alternativas de tipo constructivista, o tradicionales centradas en el profesor. Por el contrario el modelo educativo-instruccional que propone el EOS es más abierto al asumir que la optimización del aprendizaje se puede lograr con la articulación idónea de distintos tipos de configuraciones didácticas.

\section{Trabajando juntos situaciones introductorias de razonamiento proporcional en educación primaria}

En Burgos y Godino (2019) describimos la implementación del modelo instruccional de tipo mixto descrito en la sección anterior, con alumnos de educación primaria, cuyo objetivo es crearles un primer encuentro con los problemas de proporcionalidad directa e iniciar en ellos el desarrollo del razonamiento proporcional. La muestra objeto de estudio estuvo constituida por un grupo de 23 estudiantes (trece niñas y diez niños) de quinto curso (10-11 años de edad), los cuales tenían un nivel normal de desempeño en matemáticas.

En una primera configuración didáctica se presentaron a los estudiantes diversas situaciones cotidianas en las que la relación entre cantidades de dos magnitudes es de proporcionalidad directa. El precio pagado por distintas cantidades de un artículo, la distancia recorrida por un coche a velocidad constante y el tiempo. En estas situaciones, aparecen dos series de números, que se representaron en la pizarra por medio de tablas, de forma que los estudiantes podían reconocer la existencia de cierto número (la razón de proporcionalidad) que les permitía escribir cada valor de la segunda serie como producto por dicho número de los valores correspondiente de la primera serie. Se les presentó, también, algunas situaciones de no proporcionalidad, en las que los alumnos debían decidir si lo eran o no y por qué. Por ejemplo, la edad y la altura de un niño. Seguidamente la profesora - investigadora facilitó a los alumnos una hoja de trabajo con nuevas situaciones - problema introductorias. Los alumnos estaban organizados por parejas, siguiendo con la distribución habitual para trabajar en el aula con su profesor.

La segunda configuración didáctica estuvo centrada en la solución colaborativa de la tarea "Laura visita a su tío" (Figura 5). Esta tarea estaba diseñada para estimular la indagación y la discusión por medio de cuestiones dirigidas que sirvieran de acercamiento a la proporcionalidad. La resolución de esta tarea se llevó a cabo en gran grupo: los alumnos 
intervienen para completar la tabla de proporcionalidad, argumentando en cada momento la respuesta y discutiendo con los compañeros la estrategia seguida. Se siguen las recomendaciones de diversas investigaciones en relación a un primer acercamiento intuitivo al concepto de proporcionalidad, recurriendo al uso de factores multiplicativos y tablas numéricas.

Figura 5. Tarea introductoria: Laura visita a su tío

Es la fiesta fin de curso y las clases de quinto quieren encargar tartas para celebrarlo. El tío de Laura es pastelero, ¡hace unas tartas deliciosas! Así que Laura ha ido a visitarlo. Esa mañana usó 3 litros de leche para hacer 18 tartas iguales. Laura quiere saber cuántas tartas puede elaborar con 6, 2 y 5 litros de leche.

Laura, que es una chica muy lista, razona de la siguiente manera para formar una tabla como la mostrada a continuación.

- Primero, 6 es el doble de 3 (el número de litros de leche que necesitó para 18 tartas).

- Coloca tú en la tabla el número de tartas que puede hacer con 6 litros de leche.

- Luego piensa que 2 litros es la tercera parte de 6 litros. Pon el siguiente número de tartas en la tabla.

- Por último 5 litros de leche son 2 litros más los 3 litros iniciales.

Termina de llenar la tabla siguiendo estas tres ideas.

\begin{tabular}{|l|l|l|l|l|}
\hline Litros de leche & 3 & 6 & 2 & 5 \\
\hline Tartas & 18 & & & \\
\hline
\end{tabular}

¿Se te ocurre alguna forma distinta a como lo hizo Laura para completar la tabla?

Fuente: Elaborada por los autores

Al acabar cada actividad se discutieron las ideas de forma grupal, centrando la atención en el concepto de proporcionalidad y las propiedades cuyo conocimiento y comprensión se persiguen desarrollar con la tarea. Los alumnos trabajan sobre la hoja de trabajo de forma colaborativa y la profesora-investigadora podía intervenir para orientar, recordar la información necesaria y dirigir la discusión en gran grupo.

A continuación, se retomó la tarea inicial, con el objetivo de introducir la reducción a la unidad, como procedimiento para resolver una situación de proporcionalidad (Figura 6). El alumno reconoce que dadas dos magnitudes proporcionales (en este caso, el número de litros de leche y el número de tartas), conocida una de las dos cantidades de magnitud cuando la otra tiene el valor 1 , el problema es multiplicativo (tasa). 
Figura 6. Laura sigue pensando

Regresemos a la situación anterior en la que Laura estaba tratando de calcular los litros de leche que necesita su tío para hacer varias tartas. A Laura se le ocurre una idea genial (iya hemos dicho que es muy lista!): si puedo calcular el número de tartas que hace mi tío con un solo litro de leche, el cálculo para los otros litros de leche es más fácil. Para esto incluyó el 1 extra en la fila de los litros de leche:

\begin{tabular}{|l|l|l|l|l|l|}
\hline Litros de leche & 3 & 1 & 6 & 2 & 5 \\
\hline Tartas & 18 & & & & \\
\hline
\end{tabular}

Ahora, intenta responder a estas preguntas:

a) Si sabes el número de litros de leche de que dispone el pastelero, ¿de qué forma explicarías a un amigo cuantas tartas puede hacer?

b) ¿Cuántos litros de leche necesita el pastelero para hacer 4 tartas?

c) Si sabes el número de tartas que le han encargado hacer al pastelero, ¿cómo le explicarías al pastelero cuántos litros de leche necesita comprar?

Fuente: Elaborada por los autores

Después de esta sesión de trabajo colaborativo, la profesora-investigadora entregó a los alumnos una hoja de trabajo sobre las que los alumnos debían trabajar de forma personal. Se trataba de evaluar el aprendizaje logrado por medio de este modelo de introducción de la proporcionalidad.

Una de las tareas que se emplearon para evaluar los aprendizajes logrados fue la tarea del puzle de Brousseau (1997, p. 177) (Figura 7).

Figura 6. Situación del puzle propuesta a los alumnos

En la figura se presentan las piezas de un puzle. Los números escritos junto a los lados de los polígonos corresponden a las medidas de dichos lados expresadas en centímetros.

Queremos construir en cartulina este puzle, pero de mayor tamaño, de tal manera que el lado de 4 $\mathrm{cm}$ tenga una longitud de $7 \mathrm{~cm}$.

¿Sabrías que medida hay que darle a cada lado? Explica cómo lo has obtenido.

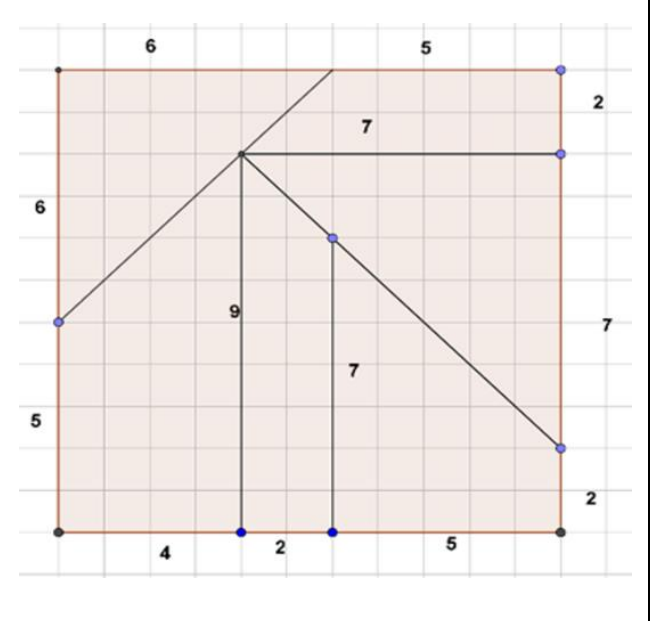

Fuente: Elaborado por los autores

La situación del puzle es parte de una secuencia de 65 lecciones experimentadas por Guy y Nadine Brousseau sobre fracciones y números decimales (Brousseau, 1997, Capítulo 4), cuyo fin es que los estudiantes construyan la única estrategia ganadora mediante una interacción con 
el medio en el que interviene el conocimiento matemático pretendido (en este caso, la proporcionalidad) que los estudiantes deben poner en juego.

En nuestra experiencia, el $70 \%$ de los estudiantes resolvieron correctamente la tarea del puzle y la estrategia de resolución predominante fue la reducción a la unidad; además, algunos estudiantes usaron estrategias de construcción progresiva (Lamon, 2007). Como mencionan Burgos y Godino (2019), parece deducirse de la aplicación de un modelo dialógicocolaborativo, que comenzar con tareas guiadas de proporcionalidad aritmética evita el uso de estrategias aditivas incorrectas reconocidas en investigaciones previas en la tarea del puzle: los estudiantes tienden de manera espontánea a construir las piezas mayores añadiendo $3 \mathrm{~cm}$ a todos los lados conocidos, ya que $7 \mathrm{~cm}$ es $3 \mathrm{~cm}$ más que $4 \mathrm{~cm}$ (Miyakawa \& Winsløw, 2009). Así, parece que este modelo de diálogo y colaboración entre el profesor y los estudiantes, en relación a la situación-problema que se pretende resolver y el contenido matemático puesto en juego, permite detectar estrategias intuitivas y aquellas que los alumnos desarrollan con poca guía por parte del profesor, aumentar el grado de implicación e interés del alumnado, e identificar conflictos semióticos y resolverlos.

\section{Reflexiones finales}

En este trabajo hemos entrado en la discusión sobre la idoneidad de distintos modelos didácticos para el aprendizaje de las matemáticas y las ciencias experimentales en la que se constata una tensión entre los modelos centrados en el estudiante y los centrados en el profesor. Hemos considerado que los primeros enfatizan el descubrimiento y la indagación autónoma del estudiante, mientras que los segundos atribuyen un papel clave a la transmisión cuando se trata del aprendizaje de contenidos nuevos para los estudiantes. Según la opinión de Kirschner et al., (2006) cuando se trata de información nueva "se debería mostrar a los aprendices qué hacer y cómo hacerlo" (p. 79). Apoyados en los supuestos antropológicos y pragmatistas sobre el conocimiento matemático y científico del EOS sostenemos que los argumentos cognitivos que apoyan la transmisión de conocimientos pueden ser complementados con razones de índole ontosemiótica, sobre todo en los momentos de "primer encuentro" del estudiante con el contenido pretendido: lo que tienen que aprender los estudiantes son, en una gran dosis, reglas epistémicas/culturales, las circunstancias de su aplicación y las condiciones requeridas para una aplicación pertinente. El aprendiz parte de reglas conocidas (conceptos, proposiciones, 
procedimientos) y produce otras, que deben ser compartidas y compatibles con las ya establecidas en la cultura matemática. Tales reglas tienen que ser almacenadas en la memoria a largo plazo del sujeto y puestas a funcionar en el momento oportuno en la memoria a corto plazo.

El postulado del aprendizaje constructivista con poca guía por parte del docente puede llevar a procesos instruccionales con baja idoneidad cognitiva y afectiva para los sujetos reales, y con baja idoneidad ecológica (adaptación al contexto) al no tener en cuenta la complejidad ontosemiótica del conocimiento matemático ni la zona de desarrollo potencial (Vygotsky, 1993) de los sujetos implicados.

Los niños no pueden descubrir las propiedades y regularidades del mundo cultural por la vía de su propia exploración independiente. Sólo pueden hacerlo por medio de la interacción y el diálogo con otros. La confianza de los niños en el testimonio, su habilidad para formular preguntas, su respeto hacia el uso de herramientas y símbolos opacos, y su selección entre informantes, avalan el hecho de que la naturaleza los ha preparado para el aprendizaje cultural (Harris, 2012, p. 267).

Consideramos que la optimización del aprendizaje implica una combinación dialéctica y compleja entre los roles del profesor como instructor (transmisor) y facilitador (gestor) y los roles del estudiante como constructor de conocimiento y receptor activo de información significativa. La idoneidad de este modelo mixto viene reforzada por la necesidad de adecuar el proyecto educativo a las restricciones temporales y la diversidad de modos y ritmos de aprendizaje en grupos de estudiantes numerosos. "Dada la miríada de situaciones potenciales de diseño, la "mejor" aproximación al diseño puede que nunca sea idéntica a ninguna otra previa, sino que verdaderamente "dependa del contexto" (Ertmer \& Newby, 1993, p. 62).

Hudson, Miller y Butler (2006) justifican la implementación de modelos instruccionales mixtos que adapten y mezclen la instrucción explícita (centrada en el profesor) con la instrucción basada en la resolución de problemas (centrada en el aprendiz) por la necesidad de realizar adaptaciones curriculares ante la diversidad de capacidades de los estudiantes. A conclusiones similares llega Steele (2005), para quien, "la mejor enseñanza a menudo integra ideas de principios constructivistas y conductistas" (p. 3).

La enseñanza de las matemáticas y de las ciencias experimentales, debe partir y centrarse en el uso de situaciones - problemas, como una estrategia para dar sentido a las técnicas y teorías estudiadas, para propiciar momentos exploratorios de la actividad matemática y desarrollar 
competencias investigativas. Sin embargo, en la práctica matemática y científica intervienen configuraciones de objetos matemáticos (conceptos, proposiciones, procedimientos, argumentos) (Font, et al., 2013), que deben ser reconocidos por el profesor para planificar su estudio. Tales objetos deben ser progresivamente dominados por los estudiantes si se desea que progresen hacia sucesivos niveles avanzados de conocimiento y competencia.

Reconocimiento: Investigación realizada como parte del proyecto de investigación PID2019105601GB-I00, con apoyo del Grupo de Investigación FQM-126 (Junta de Andalucía, Spain).

\section{Referencias}

Alfieri, L., Brooks P. J., Aldrich, N. J. \& Tenenbaum, H. R. (2011). Does discovery-based instruction enhance learning? Journal of Educational Psychology, 103(1), 1-18.

Artigue, M., \& Blomhøj, M. (2013). Conceptualizing inquiry-based education in mathematics. ZDM Mathematics Education 45, 797-810.

Baker, G. P. \& Hacker, P. M. S. (1985). Wittgenstein. Rules, grammar and necessity. An analytical commentary on the Philosophical Investigations. Glasgow: Basil Blackwell.

Ben-Chaim, D., Keret, Y. \& Ilany, B. S. (2012). Ratio and proportion: Research and teaching in mathematics teachers' education. Rotterdam: Sense Publisher

Bentley, B. \& Yates, G. C. R. (2017). Facilitating proportional reasoning through worked examples: Two classroom-based experiments. Cogent Education, 4 (1), 1297213. https://doi.org/10.1080/2331186X.2017.1297213

Bloor, D. (1983). Wittgenstein. A social theory of knowledge. London: The Macmillan Press.

Boghossian, P. (2006). Behaviorism, constructivism, and Socratic pedagogy. Educational Philosophy and Theory, 38(6), 713-722.

Breda, A., Font, V. \& Pino-Fan, L.R. (2018). Criterios valorativos y normativos en la Didáctica de las Matemáticas: el caso del constructo idoneidad didáctica. Bolema, 32 (60), p. 255 278.

Brousseau, B. (1997). Theory of didactical situations in mathematics. Dordrecht: Kluwer A. P.

Burgos, \& Godino, J. D. (2019). Trabajando juntos situaciones introductorias de razonamiento proporcional en primaria. Análisis de una experiencia de enseñanza centrada en el profesor, en el estudiante y en el contenido. Bolema, 33 (63), 389-410.

Burgos, M. \& Godino, J. D. (2020). Modelo ontosemiótico de referencia de la proporcionalidad. Implicaciones para la planificación curricular en primaria y secundaria. Avances de Investigación en Educación Matemática - AIEM (en prensa).

Ernest, P. (1994). Varieties of constructivism: Their metaphors, epistemologies, and pedagogical implications. Hiroshima Journal of Mathematics Education, 2, 1-14. 
Font, V., Godino, J. D. \& Gallardo, J. (2013). The emergence of objects from mathematical practices. Educational Studies in Mathematics, 82, 97-124.

Fox, R. (2001). Constructivism examined. Oxford Review of Education, 27 (1), 23-35.

Freudenthal, H. (1973). Mathematics as an educational task. Dordrecht: Reidel.

Freudenthal, H. (1991). Revisiting mathematics education. China lectures. Dordrecht: Kluwer.

Godino, J. D. (2013). Indicadores de la idoneidad didáctica de procesos de enseñanza y aprendizaje de las matemáticas. Cuadernos de Investigación y Formación en Educación Matemática, 11, 111-132.

Godino, J. D. (2019). How to teach mathematics and experimental sciences? Solving the inquiring versus transmission dilemma. In K. O. Villalba-Condori, A. Adúriz-Bravo, F. J. García-Peñalvo \& J. Lavonen (Eds.), Proceeding of the Congreso Internacional Sobre Educación y Tecnología en Ciencias - CISETC 2019 (Arequipa, Perú, December 10-12, 2019) (pp. 71-81). Aachen, Germany: CEUR-WS.org.

Godino, J. D. Aké, L., Gonzato, M. \& Wilhelmi, M. R. (2014). Niveles de algebrización de la actividad matemática escolar. Implicaciones para la formación de maestros. Enseñanza de las Ciencias, 32.1, 199-219.

Godino J. D., Batanero C., Cañadas G. R., \& Contreras, J. M. (2015). Linking inquiry and transmission in teaching and learning mathematics. En K. Krainer \& N. Vondrobá (Eds.), Proceedings of the Ninth Conference of the European Society for Research in Mathematics Education (CEME9, 4-8 February 2015) (pp. 2642-2648). Prague, Czech Republic: Charles University in Prague, Faculty of Education and ERME.

Godino, J. D. Batanero, C. \& Font, V. (2007). The onto-semiotic approach to research in mathematics education. ZDM. The International Journal on Mathematics Education, 39 (1-2), 127-135.

Godino, J. D., Batanero, C. \& Font, V. (2019). The onto-semiotic approach: implications for the prescriptive character of didactics. For the Learning of Mathematics, 39 (1), 37- 42.

Godino, J. D., Contreras, A. \& Font, V. (2006). Análisis de procesos de instrucción basado en el enfoque ontológico-semiótico de la cognición matemática. Recherches en Didactiques des Mathematiques, 26 (1), 39-88.

Godino, J. D., Font, V., Wilhelmi, M. R. \& Castro, C. de (2009). Aproximación a la dimensión normativa en Didáctica de la Matemática desde un enfoque ontosemiótico. Enseñanza de las Ciencias, 27(1), 59-76.

Godino, J. D., Font, V., Wilhelmi, M. R. y Lurduy, O. (2011). Why is the learning of elementary arithmetic concepts difficult? Semiotic tools for understanding the nature of mathematical objects. Educational Studies in Mathematics, 77 (2), 247-265.

Godino, J. D., Neto, T., Wilhelmi, M. R., Aké, L., Etchegaray, S. \& Lasa, A. (2015). Niveles de algebrización de las prácticas matemáticas escolares. Articulación de las perspectivas ontosemiótica y antropológica. Avances de Investigación en Educación Matemática, 8, 117-142.

Godino, J. D., Rivas, H., Burgos, M. \& Wilhelmi, M. D. (2018). Analysis of didactical trajectories in teaching and learning mathematics: overcoming extreme objectivist and 
constructivist positions. International Electronic Journal of Mathematics Education, 14(1), $147-161$

Harris, P. L (2012). The child as anthropologist. Infancia y Aprendizaje, 35 (3), 259-277.

Hmelo-Silver, C. E., Duncan, R. G., \& Chinn, C. A. (2007). Scaffolding and achievement in problem-based and inquiry learning: A response to Kirschner, Sweller, and Clark (2006). Educational Psychologist, 42(2), 99-107.

Hudson P., Miller S. P., \& Butler F. (2006). Adapting and merging explicit instruction within reform based mathematics classrooms. American Secondary Education, 35(1), 19-32

Jonassen D. H. (1991). Objectivism vs. constructivism: do we need a new philosophical paradigm? Educacional Technology Research \& Development, 39(3), 5-14.

Kirschner P. A., Sweller J., \& Clark R. E. (2006). Why minimal guidance during instruction does not work: An analysis of the failure of constructivist, discovery, problem-based, experiential, and inquiry-based teaching. Educational Psychologist, 41(2), 75-86.

Lamon, S. (2007). Rational number and proportional reasoning. Toward a theoretical framework for research. En, F. K. Lester (Ed.), Second handbook of research on mathematics teaching and learning (pp. 629-667). New York, NY: Information Age Pub Inc.

Linn, M. C., Clark, D., \& Slotta, J. D. (2003). WISE design for knowledge integration. Science Education, 87(4), 517-538.

Mayer, R. E. (2004). Should there be a three-strikes rule against pure discovery learning? American Psychologist, 59 (1), 14 - 19.

Miyakawa, T. y Winsløw, C. (2009). Didactical designs for students' proportional reasoning: an "open approach" lesson and a "fundamental situation". Educational Studies in Mathematics, 72, 199-218.

Radford, L. (2006). Introducción. Semiótica y educación matemática. Revista Latinoamericana de Matemática Educativa, Número especial, pp. 7-22.

Radford, L. (2014). De la teoría de la objetivación. Revista Latinoamericana de Etnomatemática, 7(2), 132-150.

Schoenfeld, A. H. (1992). Learning to think mathematically: Problem solving, metacognition, and sense-making in mathematics. In: D. Grouws (Ed.). Handbook of research on mathematics teaching and learning (p. 334-370). New York: MacMillan.

Simon M. A. (1995). Reconstructing mathematics pedagogy form a constructivist perspective. Journal for Research in Mathematics Education, 26, 114-145.

Simon M. A., \& Tzur R. (2004). Explicating the role of mathematical tasks in conceptual learning: An elaboration of the Hypothetical Learning Trajectory. Mathematical Thinking and Learning, 6(2), 91-104.

Steele M. M. (2005). Teaching students with learning disabilities: Constructivism of behaviorism? Current Issues in Education, 8(10). Retrieved from http://cie.ed.asu.edu/coume8/number10

Sweller J., Kirschner P. A., \& Clark R. E. (2007). Why minimally guided teaching techniques do not work: A reply to commentaries. Educational Psychologist, 42(2), 115-121. 
Thaler, R. H., \& Sunstein, C. R. (2008). Nudge improving decisions about health, wealth and happiness. Yale University Press.

Zhang, L. (2016). Is inquiry-based science teaching worth the effort? Some thoughts worth considering. Science Education, 25, 897-915.

Wilhelmi, M. R. (2017). Proporcionalidad en Educación Primaria y Secundaria. En J. M. Contreras, P. Arteaga, G. R. Cañadas, M. M. Gea, B. Giacomone y M. M. López-Martín (Eds.), Actas del Segundo Congreso International Virtual sobre el Enfoque Ontosemiótico del Conocimiento y la Instrucción Matemáticos.

Disponible en, enfoqueontosemiotico.ugr.es/civeos.html

Wittgenstein, L. (1953). Philosophical investigations. New York, NY: The MacMillan Company.

Wittgenstein, L. (1978). Remarks on the foundations of mathematics (3rd. ed.). Oxford, England: Basil Blackwell.

Vygotsky, L. S. (1993). Pensamiento y lenguaje. [Obras escogidas II, pp. 9-287]. Madrid: Visor. 